\title{
The thermal imaging camera - a new way of lipodystrophy detection in insulin treated diabetic patients
}

A. Maksymiuk-Kłos, M. Bernas, E. Jagielińska-Kalinowska, P. Piątkiewicz

Department of Internal Diseases, Diabetology and Endocrinology, Medical University of Warsaw, Warsaw, Poland

\section{Background and aims}

The number of diabetic patients treated with insulin have substantially increased in recent years, partly due to steadily growing incidence of type 1 diabetes and partly because of a common practice of the early start of using insulin in the treatment of patients with type 2 diabetes. Long term use of insulin is associated with skin related local complications like subcutaneous fat tissue lesions (lipodystrophy). It occurs in diabetic patients with a prevalence of $20-50 \%$. Lipohypertrophy is the most common cutaneous complication of insulin therapy, characterized by swelling of the fatty tissue around insulin injection sites. Subcutaneous adverse effects of insulin injections can lead to poor glycemic control. We often forget about it and look for other causes of uncontrolled glycemia.

The aim of the study was to evaluate the incidence of lipodystrophy in diabetic patients treated with insulin and to verify the effectiveness of the thermal imaging camera to detect these pathological symptoms.

\section{Materials and methods}

The analysis was conducted on the data collected from patients hospitalized at Department of Internal Diseases, Diabetology and Endocrinology of our Medical University from November 2016 to March 2017. Patients with $\mathrm{BMI} \geq 25 \mathrm{~kg} / \mathrm{m} 2$ and over 6 months insulin therapy were included in the study. We assessed $\mathrm{HbA1c}$, biochemical profile, body mass index (BMI). All patients had QuickDash and Mini Mental State Examination. Insulin administration was observed and estimated. Manual evaluation of adipose tissue and imaging with the thermal camera FLIR T 6602.0 was performed.

\section{Results}

The study included 60 patients with type 1 and 2 diabetes. $53 \%$ was male and $47 \%$ female. The duration of insulin therapy in patients ranged from 1 to 26 years. The BMI was 25 to $39 \mathrm{~kg} / \mathrm{m}^{2}$. Average $\mathrm{HbA1c}$ level was $8,02 \%$. Almost every third diabetic patient did not receive insulin properly. It was associated with greater traumatism of the tissues and worse control of diabetes ( HbAlc $>8 \%$ ) ( $p<$ 0.05 ). Subcutaneous tissue lesions were detected in $15 \%$ of patients in palpation. Using thermal imaging camera, the changes ( an example picture ) were apparent in $51 \%$ of the subjects $(p<0.05)$.

\begin{tabular}{|l|c|l|c|}
\hline Variable & \multicolumn{1}{|c|}{$n=60$} & Variable & $n=60$ \\
\hline Gender M/F \% & $53 / 47$ & LDL-C(mg/dl) & 87 (44-159) \\
\hline Age & $66,2(32-75)$ & Insulin therapy time (years) & $11(1-26)$ \\
\hline BMI (kg/m²) & $31,07(25-39)$ & Insulin dose (units) & $41(10-78)$ \\
\hline Glucose (mg/dl) & $149(79-314)$ & Incorrect insulin administration \% & 28,3 \\
\hline HbA1c\% & $8,02(5,8-14,7)$ & Palpation changes \% & 15 \\
\hline TG (mg/dl) & $185(45-469)$ & TIC changes \% & 51 \\
\hline
\end{tabular}

Table 1. Characteristics of the study group.

* TIC-Thermal Imagin Camera
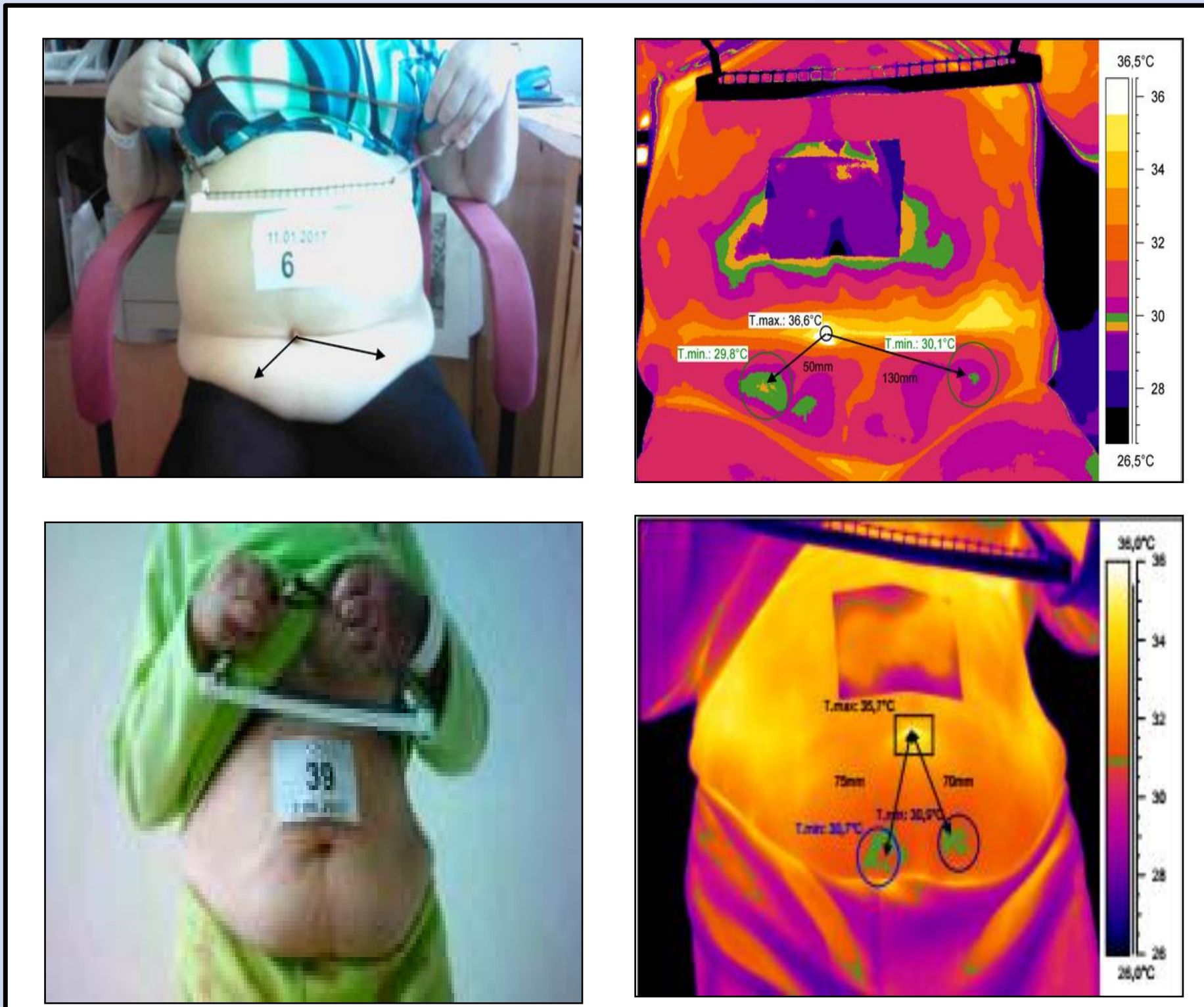

Picture 1. The sample photo from a thermal imaging camera.

\section{Conclusion}

$\square$ Lipodystrophy is an important and frequent complication of insulin therapy but is often underestimated. In our study, lipohypertrophy was present in $51 \%$ cases.

$\square$ The thermal imaging camera is a safe, inexpensive and non-invasive way to detect early changes in adipose tissue. It should be available in every Diabetology Department.

$\square$ Our study shows the need for repetitive training of diabetic patients for insulin therapy even if they have been taking insulin for a long time. It also seems to be a good direction to modify the insulin pens to facilitate the administration of drug. 